\title{
The impact of multiple irrelevant visual events at the same spatial location on inhibition
}

\author{
Troy A. W. VisSER \\ UBC Okanagan, Kelowna, British Columbia, Canada \\ AND \\ DANIEL BARNES \\ University of Melbourne, Melbourne, Victoria, Australia
}

\begin{abstract}
If an irrelevant visual event, such as a nonpredictive cue, is presented prior to a target, performance is impaired when the target appears at the cued location relative to when it is presented at an uncued location. This phenomenon, referred to as inhibition of return, can be found at multiple spatial locations when each is cued in succession. The present study examined the effect of successively cuing the same spatial location. Results suggested that additional inhibition occurred when more than one cue appeared at a single location at longer intercue intervals, but not at shorter intervals. These findings suggest that total inhibition to respond to targets at a spatial location reflects a summation of facilitatory and inhibitory factors generated by the presentation of each cue.
\end{abstract}

When a nonpredictive cue stimulus, such as a flash of light, is presented at the location of a subsequent target, responses are quicker and more accurate than when a target appears at an uncued location. Interestingly, however, as the cue-target interval increases past about $200 \mathrm{msec}$, this pattern reverses, with responses becoming slower and less accurate for cued than for uncued targets (e.g., Handy, Jha, \& Mangun, 1999; Posner \& Cohen, 1984), a pattern commonly referred to as inhibition of return (IOR). A functional role for IOR was initially proposed by Posner and Cohen, who argued that it reflected a bias against reorienting attention to a nontarget location, thereby promoting search of novel environmental locations. This theme was echoed by Klein and colleagues (e.g., Klein, 1988, 2000; Klein \& MacInnes, 1999), whose foraging facilitator hypothesis emphasized the evolutionary advantages of IOR for our ancestors' ability to find food.

An important question about the notion that IOR aids in search is whether it can be found in more than one spatial location. This is because searches typically require the examination of multiple nontargets before a target is found and, thus, maximal gains in search efficiency can be achieved only by inhibiting all or most nontarget locations. To examine this issue, Danziger, Kingstone, and Snyder (1998; see also Birmingham \& Pratt, 2005; Dodd, Castel, \& Pratt, 2003; Ogawa, Takeda, \& Yagi, 2002; Paul \& Tipper, 2003; Pratt \& Chasteen, 2007; Snyder \& Kingstone, 2000, 2001, 2007) used a cuing paradigm in which multiple cues were presented prior to target onset. On each trial in their experiment, zero to three nonpredictive cues were presented, followed by a target at either a cued or an uncued location. Danziger et al. found IOR at all cued locations, with a progressive decline in strength as cuetarget intervals increased.

Danziger et al. (1998) conclusively demonstrated that IOR occurs with multiple cues presented at different spatial locations, but they did not consider the issue of how multiple cues at a single location might influence IOR. Such an experiment would be a simulation of a frequent real-life scenario in which visual events that would normally capture attention occur repeatedly at a single spatial location. For example, while standing in line at a movie theatre, a stranger may repeatedly wave to someone behind you in the line. This salient visual event would presumably draw attention and then inhibition. The critical question is whether inhibition would increase with the stranger's continued waving and, thus, with the additional impetus for attending to the stranger's location.

Given that cues at different locations each yield IOR (Danziger et al., 1998), it is reasonable to conjecture that multiple cues at the same location would do the same. On this option, for example, two cues presented at a single location should produce more IOR than would a single cue. Pratt and Abrams (1995) examined this issue directly by presenting to observers two possible target locations flanking a central fixation. On each trial, two cues were displayed: They could occur at the same target location, at different target locations, or at one target location and the central fixation. The results from this study were equivocal. On one hand, more IOR occurred when a target loca-

T. A.W.Visser, t.visser@uq.edu.au 
tion was cued twice, supporting the suggestion that multiple cues at the same location do lead to increased IOR. On the other hand, response times (RTs) were identical for targets appearing at an uncued location and for those appearing at the location of the first of the two cues, implying that the appearance of a second cue nullified the inhibition generated by the first cue - a result inconsistent with the suggestion that two cues at the same location should generate additional inhibition.

In a later study, Tipper, Weaver, and Watson (1996) criticized the ecological validity of Pratt and Abrams's (1995) work on the grounds that it employed only two possible target locations; when two different locations were cued, participants were essentially required to inhibit all possible target locations. In support of number of locations as a critical factor, Tipper et al. presented to observers four possible target locations and three consecutive cues and showed inhibition for targets presented at all three cued locations. They also demonstrated a trend toward increasing inhibition across cues, consistent with Danziger et al.'s (1998) work and consistent with the notion that multiple cues in the same location should lead to greater IOR. For the present purposes, however, it is important that Danziger et al. did not present two cues at the same location. Thus, their work does not comment directly on our research question.

Finally, in a response to Tipper et al. (1996), Abrams and Pratt (1996) showed that whereas multiple spatially adjacent cues each yielded IOR, the effect was greatly ameliorated when cued locations were not adjacent. That said, as Tipper et al. pointed out, there was still a trend toward greater IOR with increasing numbers of cues in the majority of conditions run by Abrams and Pratt. In summary, although the one previous study to present multiple cues in the same location (Pratt \& Abrams, 1995) showed more IOR than when a single cue was presented, conflicting results from that study and from similar follow-up experiments (Abrams \& Pratt, 1996; Tipper et al., 1996) make interpretation of this finding uncertain.

Another study relevant to the issue of how multiple cues presented at the same spatial location can influence IOR was done by Maylor and Hockey (1987), who found that more IOR occurred on trials where both the cue and the preceding target appeared at the same location as a target. This finding implied that orienting attention to a location twice (preceding target, then cue) yielded more IOR than did orienting attention only once (cue only). Consistent with this, Maylor and Hockey also found that target RTs increased as a function of the number of consecutive trials on which the target appeared in the same spatial location.

Although consistent with the findings of Pratt and Abrams (1995), Maylor and Hockey's (1987) results do not directly address the issue of how multiple irrelevant events (i.e., cues) influence IOR. In both teams' experiments, additional IOR was obtained only when targets appeared at the location of a prior target. This opens up the possibility that the effect depends in some fashion on a prior relevant stimulus appearing at a spatial location. Also potentially problematic is that, as in Pratt and
Abrams, there were only two possible target locations in Maylor and Hockey's experiments. Taken together, these issues make it difficult to predict a priori from Maylor and Hockey's paradigm how strong inhibition should be following longer sequences of irrelevant cues.

To address these concerns, we used a modified version of the multiple-cuing paradigm employed by Danziger et al. (1998). In the present Experiment 1, observers were presented a four-location search matrix centered on a fixation cross located in the middle of the display. Each search location was demarcated by a placeholder box. On each trial, two cues and a single target were presented at random locations. Thus, on some trials, cues occurred in two different locations; on others, they occurred in the same location. This procedure allowed us to replicate the results of Danziger et al., thereby validating our paradigm, and to evaluate the impact of presenting two cues at a single location relative to presenting only a single cue.

\section{EXPERIMENT 1}

\section{Method}

Participants. Twenty-four participants ( 16 female) were recruited through advertisements on University of Melbourne notice boards. Informed consent was obtained from all participants per standard ethical guidelines. All participants received a small honorarium of A $\$ 10$ to compensate them for their time and effort. All participants reported normal or corrected-to-normal vision and were naive about the purpose of the experiment.

Apparatus and Stimuli. All stimuli were presented on a 19in. Viewsonic monitor (Model G190T) running at a refresh rate of $100 \mathrm{~Hz}$ and slaved to a Pentium 4 computer running Presentation software (Version 9.20; Neurobehavioral Systems, 2005). The software was also responsible for recording RTs and accuracy from a computer keyboard.

Testing was conducted in a quiet, dark laboratory with only dim illumination of the keyboard provided by a small light. The viewing distance from the monitor was approximately $60 \mathrm{~cm}$. For the duration of each trial, observers viewed a display consisting of four outlined gray squares $\left(2^{\circ} \times 2^{\circ}\right.$; luminance $\left.=27 \mathrm{~cd} / \mathrm{m}^{2}\right)$. These placeholder squares formed an imaginary cross centered on a fixation cross $\left(0.3^{\circ} \times 0.3^{\circ}\right.$; luminance $\left.=27 \mathrm{~cd} / \mathrm{m}^{2}\right)$, with each square being equidistant from fixation. The center of each placeholder was $6^{\circ}$ from fixation. Cues consisted of the brightening of a square to $114 \mathrm{~cd} / \mathrm{m}^{2}$ and an increase in square thickness from 4 to 6 pixels. Targets consisted of a solid luminous square $\left(1.2^{\circ} \times 1.2^{\circ}\right.$; luminance $=$ $114 \mathrm{~cd} / \mathrm{m}^{2}$ ) presented at the center of a placeholder. All stimuli were presented against a black background.

Procedure. Trials were divided so that an equal number of first cues, second cues, and targets appeared at each of the four possible locations. This complete counterbalancing yielded a block of 64 trials. The entire experiment comprised 11 blocks, for a total of 704 trials.

Prior to beginning the experiment, observers were given written and oral instructions that stressed two points: Observers should keep their eyes centered at fixation, and cues would not predict target location and, therefore, should be ignored. Each trial began with the presentation of a fixation cross flanked by four outline squares that served as placeholders. These placeholders remained on the display for the duration of the trial. Observers were instructed to press the space bar to start the sequence of stimuli, at which point the fixation cross disappeared. Following a pause of $500 \mathrm{msec}$, the first cue was presented for $100 \mathrm{msec}$. The cue disappeared, and a second pause occurred for $700 \mathrm{msec}$. This yielded a stimulus onset asynchrony (SOA) of $800 \mathrm{msec}$ between cues. Then, the second cue was presented for $100 \mathrm{msec}$. 
Finally, after another 700-msec pause, the target was presented for $1,000 \mathrm{msec}$ or until a response was made by pressing the space bar. This yielded an $800-$ msec SOA between the second cue and target. On $12.5 \%$ of the trials (divided equally between blocks), no target was presented. These catch trials were designed to discourage participants from anticipating target onset. To aid in this, we presented feedback at the end of each block that informed observers about their accuracy on the catch trials. If they were found to have made many errors, they were encouraged to slow down and be more careful.

\section{Results}

Each observer's first 30 trials were treated as practice and were omitted from further analysis. For the purposes of data analysis, following the model of Danziger and colleagues (e.g., Danziger et al., 1998), the trials were divided into the following types: (1) catch trials, on which no target was presented; (2) double-cued trials, on which both cues and the target appeared at the same location; (3) oneback trials, on which cues appeared at different locations, with the target appearing at the location of the second cue; (4) two-back trials, on which cues appeared at different locations, with the target appearing at the location of the first cue; (5) uncued-single trials, on which cues appeared at different locations and the target appeared at an uncued location, and (6) uncued-double trials, on which cues appeared at the same location and the target appeared at an uncued location.

Catch-trial errors were defined as responses made when no target was present. The mean error rate (ER) on catch trials was $3.62 \%$, indicating that observers generally complied with task instructions and waited for target onset before responding. Errors on all other trial types were defined as responses made less than $200 \mathrm{msec}$ or more than 1,000 msec after target onset. ERs across conditions were quite low $(1.14 \%-1.95 \%)$, indicating that observers neither anticipated nor missed many targets. Analysis of ERs revealed no significant differences across conditions $\left[F(4,92)=1.99, p>.10, \eta^{2}=.080\right]$.

Only trials on which no errors were made were included in the remainder of the analyses. Mean RTs on these trials were calculated separately for the two-back, one-back, double-cued, uncued-single, and uncued-double conditions. These means are illustrated in Figure 1 and are separated on the basis of whether targets appeared at a cued or an uncued location.

We first compared RTs in appropriate cued and uncued conditions in order to determine whether IOR occurred. It should be noted that in this and all future analyses, both the two-back and one-back conditions are compared with the same uncued-single baseline condition. RTs in the two-back condition were slower than those in the uncued-single condition $[t(23)=6.81, p<.001]$, RTs in the one-back condition were slower than those in the uncued-single condition $[t(23)=10.43, p<.001]$, and RTs in the double-cued condition were slower than those in the uncued-double condition [t(23) $=8.06, p<.001]$, indicating that significant IOR had occurred in all three cases.

To compare the magnitude of IOR in each cuing condition, we next calculated difference scores between appro-

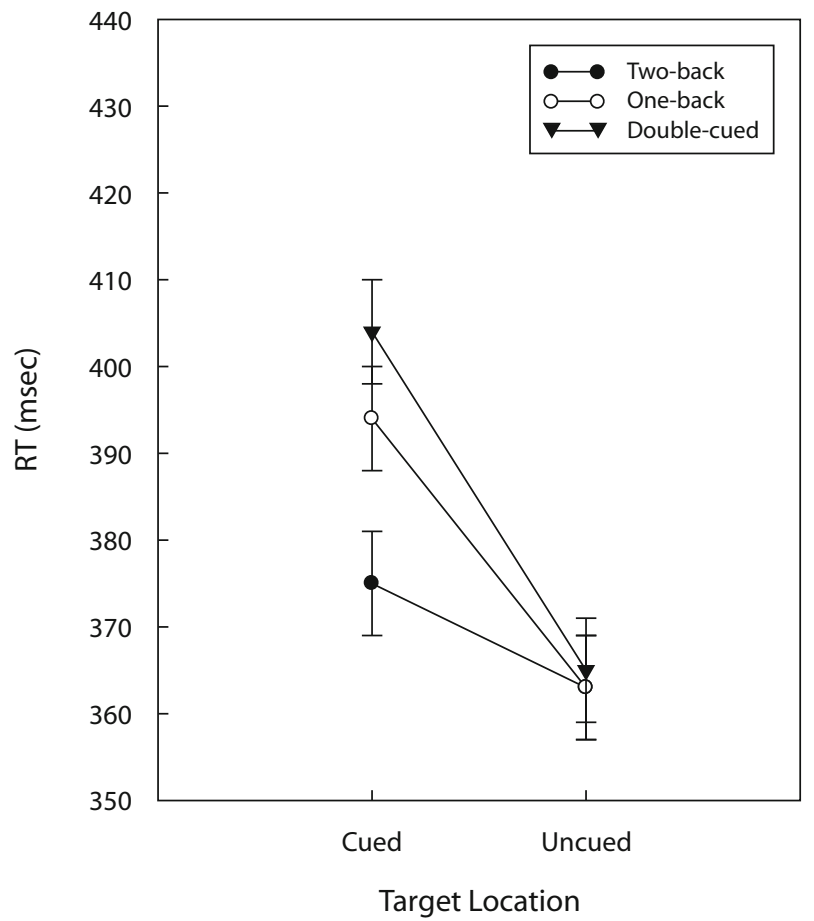

Figure 1. Mean target response times (RTs, in milliseconds) on correct trials, as a function of whether targets were presented at cued or uncued locations in Experiment 1. "Two-back" refers to trials on which targets appeared in the first location cued; "oneback" refers to trials on which targets appeared in the second location cued; "double-cued" refers to trials on which targets appeared at a location cued twice. Note that two-back and oneback uncued trials are identical. Error bars represent withinparticipants $95 \%$ confidence intervals calculated using the methodology outlined by Masson and Loftus (2003).

priate cued and uncued conditions. This yielded IOR magnitudes of $12 \mathrm{msec}$ in the two-back condition, $31 \mathrm{msec}$ in the one-back condition, and $39 \mathrm{msec}$ in the double-cued condition. These means were then submitted to a one-way ANOVA, which indicated a significant difference across cue conditions $\left[F(2,46)=29.14, p<.001, \eta^{2}=.559\right]$. LSD post hoc comparisons revealed significant differences between the one-back and two-back conditions $(p<.001)$, the two-back and double-cued conditions $(p<.001)$, and most important, the one-back and doublecue conditions $(p<.04)$. This last comparison indicated that, given the same cue-target interval $(800 \mathrm{msec})$, significantly more IOR occurred at a location that had been cued twice than at a location that had been cued once.

The results of Experiment 1 indicate that robust IOR occurred at multiple cued locations. This replicates the earlier finding of Danziger et al. (1998; Snyder \& Kingstone, $2000,2001,2007)$ and validates that the conditions in our experimental paradigm led to significant IOR. It is also consistent with the view that IOR can boost the efficiency of visual search by inhibiting multiple nontarget locations. Most critical for our present purposes, Experiment 1 also suggests that cuing the same location twice yields more IOR than does cuing it only once. This implies that the repetition of an irrelevant visual event leads to inhibition, 
regardless of whether that event occurs at a novel location or at the location of a previous irrelevant event. These results are consistent with those of Pratt and Abrams (1995), as well as with those of Maylor and Hockey (1987), and suggest that multiple relevant or irrelevant visual events both yield increased IOR, even in paradigms with more than two possible target locations.

Before drawing strong conclusions, however, it is important to consider whether our findings are peculiar to our particular paradigm. In particular, in the present experiment, the fixation cross disappeared from the display once observers initiated a trial. This could have increased the likelihood of observers' eyes' wandering toward cued locations, particularly during double-cue trials, and could have influenced the results obtained here. Also of interest is whether our results are peculiar to the number of possible target locations and their spatial configuration. To examine these issues, in Experiment 2, the fixation cross remained present throughout a trial, and we changed the number of possible target locations and their spatial configuration.

\section{EXPERIMENT 2}

\section{Method}

Participants. Thirty participants (20 female) were recruited through advertisements on UBC Okanagan notice boards or signed up through Web-based software. Informed consent was obtained from all participants per standard ethical guidelines. All participants received a small honorarium of $\mathrm{C} \$ 10$ or bonus credit toward their grade in a psychology course to compensate them for their time and effort. All participants reported normal or corrected-to-normal vision, and none had participated in the previous experiment.

Apparatus and Stimuli. The apparatus and stimuli were identical to those used in Experiment 1, except that stimuli were presented on a 19-in. NEC monitor (MultiSync FE992).

Procedure. The procedure was identical to that used in Experiment 1, with the following exceptions: (1) The fixation cross remained on the display throughout the experiment; (2) rather than having participants press the space bar to initiate a trial, each trial in a block ran automatically, with the beginning of each new trial signaled by the fixation cross's changing color from gray to red for $200 \mathrm{msec}$, and then returning to gray; and (3) the number of possible target locations was reduced from four to three, with the placeholder boxes forming an imaginary equilateral triangle centered at fixation.

The center-to-center separation between the fixation cross and each placeholder box was approximately $5^{\circ}$. We opted to decrease the number of locations (rather than increase them) because we wanted to counterbalance the location of first and second cues, as well as of the target, so that each of these items appeared once at each location in each block of trials. However, with five or more locations, this aspect of our design would have required a prohibitively large number of trials in each block in order to attain an acceptable level of statistical power. The experiment consisted of 810 trials divided into 30 blocks of 27 trials. Each block included 3 catch trials on which no target was presented, yielding a catch-trial frequency of $11.1 \%$.

\section{Results}

The first block of 27 trials from each observer was treated as practice and was omitted from data analysis. The mean ER on catch trials was $4.18 \%$, indicating that observers complied with task instructions. As in Experiment 1, mean ERs were calculated separately for trials in the two-back, one-back, double-cued, uncued-single, and uncued-double conditions. These rates were quite low $(2.45 \%-3.16 \%)$, indicating that observers neither anticipated nor missed many targets. Analysis of ERs revealed no significant differences across conditions $[F(4,116)=$ $\left.0.92, p>.45, \eta^{2}=.031\right]$.

Only trials on which no errors were made were included in the remainder of analyses. Mean RTs on these trials were calculated separately for the two-back, one-back, double-cued, uncued-single, and uncued-double conditions. These means are illustrated in Figure 2 and are separated on the basis of whether the targets had appeared at cued or uncued locations.

Consistent with those in the presence of IOR, RTs in the two-back condition were slower than those in the uncued-single condition $[t(29)=7.57, p<.001]$, RTs in the one-back condition were slower than those in the uncued-single condition $[t(29)=9.35, p<.001]$, and RTs in the double-cued condition were slower than those in the uncued-double condition $[t(29)=10.78, p<$ $.001]$.

In order to compare the magnitude of IOR in each cuing condition, we calculated difference scores between appropriate cued and uncued conditions. This yielded IOR mag-

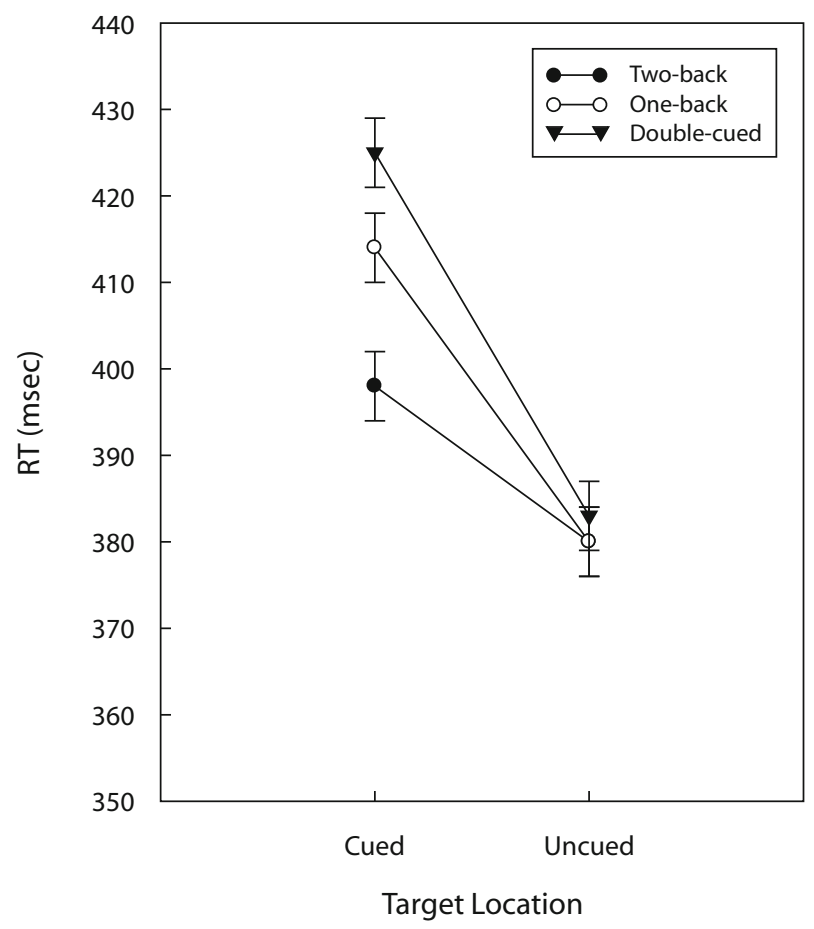

Figure 2. Mean target response times (RTs, in milliseconds) on correct trials, as a function of whether targets were presented at cued or uncued locations in Experiment 2. "Two-back" refers to trials on which targets appeared in the first location cued; "oneback" refers to trials on which targets appeared in the second location cued; "double-cued" refers to trials on which targets appeared at a location cued twice. Note that two-back and oneback uncued trials are identical. Error bars represent withinparticipants $95 \%$ confidence intervals calculated using the methodology outlined by Masson and Loftus (2003). 
nitudes of $18 \mathrm{msec}$ in the two-back condition, $34 \mathrm{msec}$ in the one-back condition, and $42 \mathrm{msec}$ in the double-cued condition. These means were then submitted to a one-way ANOVA, which indicated a significant difference across cue conditions $\left[F(2,58)=38.31, p<.001, \eta^{2}=.569\right]$. LSD post hoc comparisons revealed significant differences between the one-back and two-back conditions $(p<.001)$ and between the two-back and double-cued conditions $(p<.001)$. Finally, as in Experiment 1 , there was a highly significant difference between the one-back and double-cued conditions $(p<.01)$, indicating that given the same cue-target interval ( $800 \mathrm{msec})$, significantly more IOR occurred at locations cued twice than at locations cued once.

In summary, the results of Experiment 2 replicated the principal findings of Experiment 1, including the presence of multiple-location IOR and the finding that IOR increased at locations cued on multiple occasions. As well as replicating the general pattern of differences between cue conditions, the magnitude of IOR in the one-back, two-back, and double-cued conditions was also quite similar between experiments. In fact, the only difference of note between Experiments 1 and 2 was that overall RTs were about $15 \mathrm{msec}$ slower at both cued and uncued locations - a difference that seems to have been replicated in the subsequent experiments and thus was likely due to changes in participant population between Experiment 1 and the subsequent experiments.

The broad similarities between Experiments 1 and 2 suggest that our results are not due to disappearance of fixation, number of potential target locations, or their spatial configuration. Instead, the option must be considered that differences in the magnitude of IOR between singleand double-cued locations reflect the cumulative effects of each cue on target detection. It seems that presentation of a cue, whether at a previously cued location or not, results in resources being allocated to that location, resulting in a period of relatively enhanced target responses, followed by inhibition resulting in relatively impaired target responses. When this occurs at a location that has already been cued, the effects of the new cue are summated with those arising from previous cues.

Although this explanation provides a concise account of the enhanced IOR found at double-cued locations in Experiments 1 and 2, it is desirable to test whether it can be used to predict the outcome of other experimental manipulations. Two such predictions are that when the SOA between the second cue and target is brief (100 msec), (1) less IOR should be found at double-cued locations than when the second cue-target interval is longer (e.g., $800 \mathrm{msec}$ ), and (2) less IOR should be found at doublecued locations than at two-back locations, although the SOA between the cue and target is nearly identical. Both of these predictions follow from the notion that at the 100 -msec second cue-target SOA, the second cue will attract processing resources, thereby improving detection ability. Per the first prediction, this benefit should ameliorate IOR relative to the $800-\mathrm{msec}$ SOA, when the second cue will generate additional inhibition. Similarly, per the second prediction, at the 100-msec SOA, less IOR would be expected at double-cued locations than at the one-back locations because double-cued locations receive the beneficial influence of the second cue, whereas oneback locations do not. These predictions were evaluated in Experiment 3.

\section{EXPERIMENT 3}

\section{Method}

Participants. Twenty-seven participants ( 24 female) were recruited through advertisements on UBC Okanagan notice boards or signed up through Web-based software. Informed consent was obtained from all participants per standard ethical guidelines. All participants received a small honorarium of $\mathrm{C} \$ 10$ or bonus credit toward their grade in a psychology course to compensate them for their time and effort. All participants reported normal or corrected-to-normal vision, and none had participated in the previous experiments.

Apparatus and Stimuli. The apparatus and stimuli were identical to those used in Experiment 2.

Procedure. The procedure was identical to that used in Experiment 2, except that the trials were equally divided among three different second cue-target SOAs: 100, 400, and $800 \mathrm{msec}$. Thus, the longest SOA was equivalent to that used in Experiments 1 and 2. In order to completely counterbalance all trial types across the three different SOAs, participants completed 11 blocks of 81 trials, totaling 891 trials. Each block included 9 catch trials, on which no target was presented, yielding a catch-trial frequency of $11.1 \%$.

\section{Results}

The first 30 trials from each observer were treated as practice and were omitted from data analysis. On catch trials, the interval between the offset of the second cue and the end of a trial varied with second cue-target SOA. Thus, mean ERs were calculated separately at the 100-, 400-, and 800-msec intervals. These ERs were 2.08\%, $1.01 \%$, and $2.14 \%$, respectively, and did not differ as a function of second cue-target SOA $[F(2,52)=2.61, p>$ $\left..08, \eta^{2}=.091\right]$.

Mean ERs were calculated separately at each second cue-target SOA for trials in the two-back, one-back, double-cued, uncued-single, and uncued-double conditions. These rates were quite low $(0.67 \%-2.04 \%)$, indicating that observers neither anticipated nor missed many targets. To examine the impact of second cue-target SOA on ERs in the two-back condition, we submitted relevant means to a 3 (SOA: 100, 400, $800 \mathrm{msec}$ ) $\times 2$ (validity: cued, uncued) within-participants ANOVA. This analysis revealed no significant main effects or interactions $\left(F \mathrm{~s}<1.08, p \mathrm{~s}>.34, \eta^{2} \mathrm{~s}<.041\right)$. An identical analysis on ERs in the one-back condition revealed a significant main effect of SOA, with ERs declining as SOA increased $\left[F(2,52)=3.38, p<.05, \eta^{2}=.115\right]$. No other main effects or interactions were significant $(F \mathrm{~s}<0.90, p \mathrm{~s}>.41$, $\left.\eta^{2} \mathrm{~s}<.034\right)$. Finally, an identical analysis on ERs in the double-cued condition revealed a significant interaction $\left[F(2,52)=3.17, p=.05, \eta^{2}=.109\right]$. Examination of the error data did not show a clearly interpretable pattern underlying this interaction, with ERs being slightly lower in the valid condition than in the invalid condition at the 100-msec SOA, slightly higher in the valid condition than in the invalid condition at the 400-msec SOA, and nearly 
equal at the $800-\mathrm{msec}$ SOA. The main effects were not significant $\left(F_{\mathrm{s}}<2.32, p \mathrm{~s}>.10, \eta^{2} \mathrm{~s}<.082\right)$.

Only trials on which no errors were made were included in the remainder of analyses. Mean RTs on these trials were calculated separately at each second cue-target SOA for trials in the two-back, one-back, double-cued, uncuedsingle, and uncued-double conditions. These means are illustrated in Figure 3 and are separated on the basis of SOA and whether targets had appeared at cued or uncued locations. The presentation of the data in this way allows easy comparison with the results of the previous two experiments, although it should be noted that analyses reported below are for specific conditions compared across the SOAs, as opposed to between different conditions within a single SOA.

To examine the impact of second cue-target SOA in the two-back condition, we submitted relevant means to a 3 (SOA) $\times 2$ (validity) within-participants ANOVA. This revealed significant main effects of SOA $[F(2,52)=$ $\left.61.11, p<.001, \eta^{2}=.702\right]$ and validity $[F(1,26)=$ $\left.37.69, p<.001, \eta^{2}=.592\right]$, but no interaction between these factors $\left[F(2,52)=0.07, p>.93, \eta^{2}=.003\right]$. The main effect of SOA reflects a steady decline in overall RTs with an increase in SOA. This change may reflect increased target preparedness as the interval between the second cue and target increased (Fernandez-Duque \& Posner, 1997; Posner, 1980) or, alternatively, may reflect a recovery from response inhibition, which likely occurred at the shortest SOA due to the rapid successive presentation of the cue (which was to be ignored) and the target (Coward, Poliakoff, O'Boyle, \& Lowe, 2004; Gellatly, Cole, Fox, \& Johnson, 2003; Harvey, 1980). To examine the main effect of validity, we compared RTs for cued and uncued targets at each SOA. These comparisons revealed IOR magnitudes of $18 \mathrm{msec}$ at the 100 -msec SOA $[t(26)=5.55, p<.001], 17 \mathrm{msec}$ at the 400 -msec SOA $[t(26)=4.87, p<.001]$, and $16 \mathrm{msec}$ at the $800-\mathrm{msec}$ SOA $[t(26)=3.50, p<.01]$. This is consistent with the results of the previous two experiments and suggests that the magnitude of IOR in the two-back condition remained relatively constant across cue-target intervals ranging from 800 to $1,600 \mathrm{msec}$.

To examine the impact of second cue-target SOA in the one-back condition, we submitted the relevant means to a $3(\mathrm{SOA}) \times 2$ (validity) within-participants ANOVA. This revealed significant main effects of SOA $[F(2,52)=$ $\left.26.84, p<.001, \eta^{2}=.508\right]$ and validity $[F(1,26)=27.45$, $\left.p<.001, \eta^{2}=.514\right]$, as well as an interaction between these factors $\left[F(2,52)=39.03, p<.001, \eta^{2}=.600\right]$. As in the two-back condition, the main effect of SOA reflects the fact that overall RTs declined as SOA increased. To examine the interaction, we compared RTs for cued and uncued targets at each SOA. These comparisons revealed a facilitatory effect of $7 \mathrm{msec}$ at the 100 -msec SOA $[t(26)=$ $1.72, p<.05$, one-tailed] and IOR magnitudes of $25 \mathrm{msec}$ at the $400-\mathrm{msec} \operatorname{SOA}[t(26)=7.31 p<.001]$ and $31 \mathrm{msec}$ at the $800-\mathrm{msec} \mathrm{SOA}[t(26)=6.88, p<.001]$. This pattern of facilitation followed by inhibition with increasing SOA suggests that when the second cue was presented in a previously uncued location, it generated a period of facilitation followed by inhibition, as is common in studies where a single nonpredictive cue is followed by a target (e.g., Posner \& Cohen, 1984).

Finally, to examine the impact of second cue-target SOA in the double-cued condition, we submitted the relevant means to a $3(\mathrm{SOA}) \times 2$ (validity) within-participants

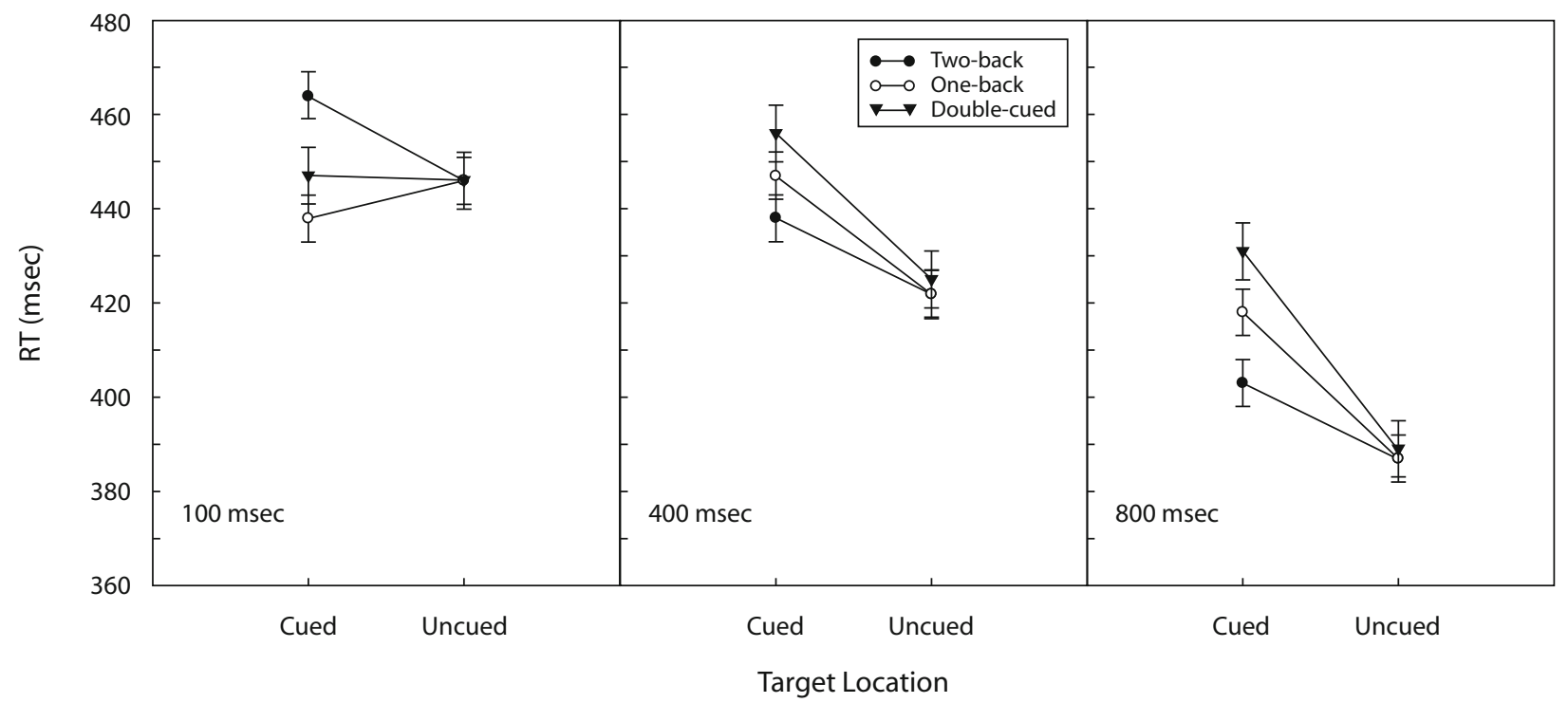

Figure 3. Mean target response times (RTs, in milliseconds) on correct trials, as a function of whether targets were presented at cued or uncued locations in Experiment 3. Numbers in the bottom-left corner of each graph represent the second cue-target SOA for that condition. "Two-back" refers to trials on which targets appeared in the first location cued; "one-back" refers to trials on which targets appeared in the second location cued; "double-cued" refers to trials on which targets appeared at a location cued twice. Note that two-back and one-back uncued trials are identical. Error bars represent within-participants $95 \%$ confidence intervals calculated using the methodology outlined by Masson and Loftus (2003). 
ANOVA. This revealed significant main effects of SOA $\left[F(2,52)=21.61, p<.001, \eta^{2}=.454\right]$ and validity $\left[F(1,26)=42.95, p<.001, \eta^{2}=.623\right]$, as well as an interaction between these factors $[F(2,52)=21.49, p<$ $\left..001, \eta^{2}=.453\right]$. As in the one-back and two-back conditions, the main effect of SOA indicates that overall RTs declined as SOA increased. To examine the interaction, we compared RTs for cued and uncued targets at each SOA. These comparisons revealed a nonsignificant difference of $1 \mathrm{msec}$ at the $100-\mathrm{msec}$ SOA $[t(26)=0.02, p>.05]$ and IOR magnitudes of $30 \mathrm{msec}$ at the $400-\mathrm{msec}$ SOA $[t(26)=5.88, p<.001]$ and $43 \mathrm{msec}$ at the 800 -msec SOA $[t(26)=7.37, p<.001]$.

The purpose of Experiment 3 was to test the hypothesis that presenting a second cue at a previously cued location attracts processing resources, followed by inhibition, and that these effects combine with those generated by prior cues. This account makes two predictions concerning the results of the present study. First, at the 100-msec SOA, less IOR should be found at the double-cued location than at the 800 -msec SOA. We tested this by comparing difference scores between cued and uncued targets at the 100and 800 -msec SOAs. The results supported the prediction, with significantly less IOR at the 100 -msec SOA $[t(26)=$ $6.44, p<.001]$. The second prediction of this account was that less IOR should be found at double-cued locations than at two-back locations at the 100-msec SOA, reflecting the fact that presentation of the second cue attracts resources, thereby facilitating responses. To test this prediction, we compared difference scores between cued and uncued targets in the double-cued condition and twoback conditions. Again the results supported the prediction, with less IOR in the double-cued condition $[t(26)=$ $3.68, p<.01]$.

In keeping with the analyses of previous experiments, we also compared the magnitude of facilitation/IOR between the double-cued and one-back conditions at each second cue-target SOA. At the 100-msec SOA, there was significantly more facilitation in the one-back condition $[t(26)=2.67, p<.02]$. This is consistent with the notion that the inhibitory effects of the prior cue in the doublecued condition slowed target responses. At the 400 -msec SOA, there was a nonsignificant trend toward greater IOR in the double-cued condition $[t(26)=1.02, p>.31]$. Finally, at the 800 -msec SOA, which was identical to that in Experiments 1 and 2, there was significantly greater IOR in the double-cued condition $[t(26)=2.51, p<.02]$. Taken together, these findings replicate those of the previous two experiments and are consistent with the notion that cuing a location twice results in a summation of the effects of both cues on target detection.

The goals of Experiment 4 were twofold. First, we wanted to determine whether the influence of double cuing a location would extend to tasks other than simple detection. Second, given the theoretical importance of the pattern of findings obtained in Experiment 3, we wished to replicate the results using a different paradigm. To these ends, in Experiment 4, we changed the target-detection task to a target-localization task.

\section{EXPERIMENT 4}

\section{Method}

Participants. Twenty-four participants ( 18 female) were recruited through advertisements on UBC Okanagan notice boards or signed up through Web-based software. Informed consent was obtained from all participants per standard ethical guidelines. All participants received a small honorarium of $\mathrm{C} \$ 10$ or bonus credit toward their grade in a psychology course to compensate them for their time and effort. All participants reported normal or corrected-to-normal vision, and none had participated in the previous experiments.

Apparatus and Stimuli. Apparatus and stimuli were identical to those used in Experiment 3.

Procedure. The procedure was identical to that used in Experiment 3, with one exception: Participants were instructed to report the location of the target by pressing the up arrow key if the target appeared at the top location, the left arrow key if the target appeared at the bottom-left location, and the right arrow key if the target appeared at the bottom-right location.

\section{Results}

The first 30 trials from each observer were treated as practice and were omitted from data analysis. The mean ERs on catch trials were $0.63 \%, 0.41 \%$, and $0.57 \%$ at the $100-, 400-$, and 800-msec second cue-target SOAs, respectively. Analysis of these ERs revealed no significant difference among these values $[F(2,46)=0.27, p>.76$, $\left.\eta^{2}=.012\right]$.

Mean ERs were calculated separately at each second cue-target SOA for trials in the two-back, one-back, double-cued, uncued-single, and uncued-double conditions. These rates were low $(2.44 \%-6.23 \%)$, indicating that observers neither anticipated nor inaccurately localized many targets. To examine the impact of second cue-target SOA on ERs in the two-back condition, we submitted relevant means to a 3 (SOA) $\times 2$ (validity) within-participants ANOVA. This analysis revealed a significant main effect of SOA, with ERs declining as SOA increased $[F(2,46)=$ $\left.3.37, p<.05, \eta^{2}=.128\right]$. No other main effects or interactions were significant $\left(F_{\mathrm{s}}<0.65, p \mathrm{~s}>.43, \eta^{2} \mathrm{~s}<\right.$ $.028)$. An identical analysis of ERs in the one-back condition revealed a main effect of SOA, with ERs declining as SOA increased $\left[F(2,46)=4.69, p<.02, \eta^{2}=.169\right]$. No other main effects or interactions were significant $\left(F_{\mathrm{S}}<\right.$ $\left.0.93, p \mathrm{~s}>.35, \eta^{2} \mathrm{~s}<.040\right)$. Finally, analysis of ERs in the double-cued condition revealed a main effect of SOA, with ERs declining as SOA increased $[F(2,46)=3.31, p<$ $\left..05, \eta^{2}=.126\right]$. No other main effects or interactions were significant $\left(F \mathrm{~s}<2.82, p \mathrm{~s}>.069, \eta^{2} \mathrm{~s}<.011\right)$.

Only trials on which no errors were made were included in the remainder of the analyses. Mean RTs on these trials were calculated separately at each second cue-target SOA for the two-back, one-back, double-cued, uncuedsingle, and uncued-double conditions. These means are illustrated in Figure 4 and are separated on the basis of SOA and whether targets had appeared at cued or uncued locations.

To examine the impact of second cue-target SOA in the two-back condition, we submitted relevant means to a 3 (SOA: $100,400,800 \mathrm{msec}) \times 2$ (validity: cued, uncued) within-participants ANOVA. This revealed significant 


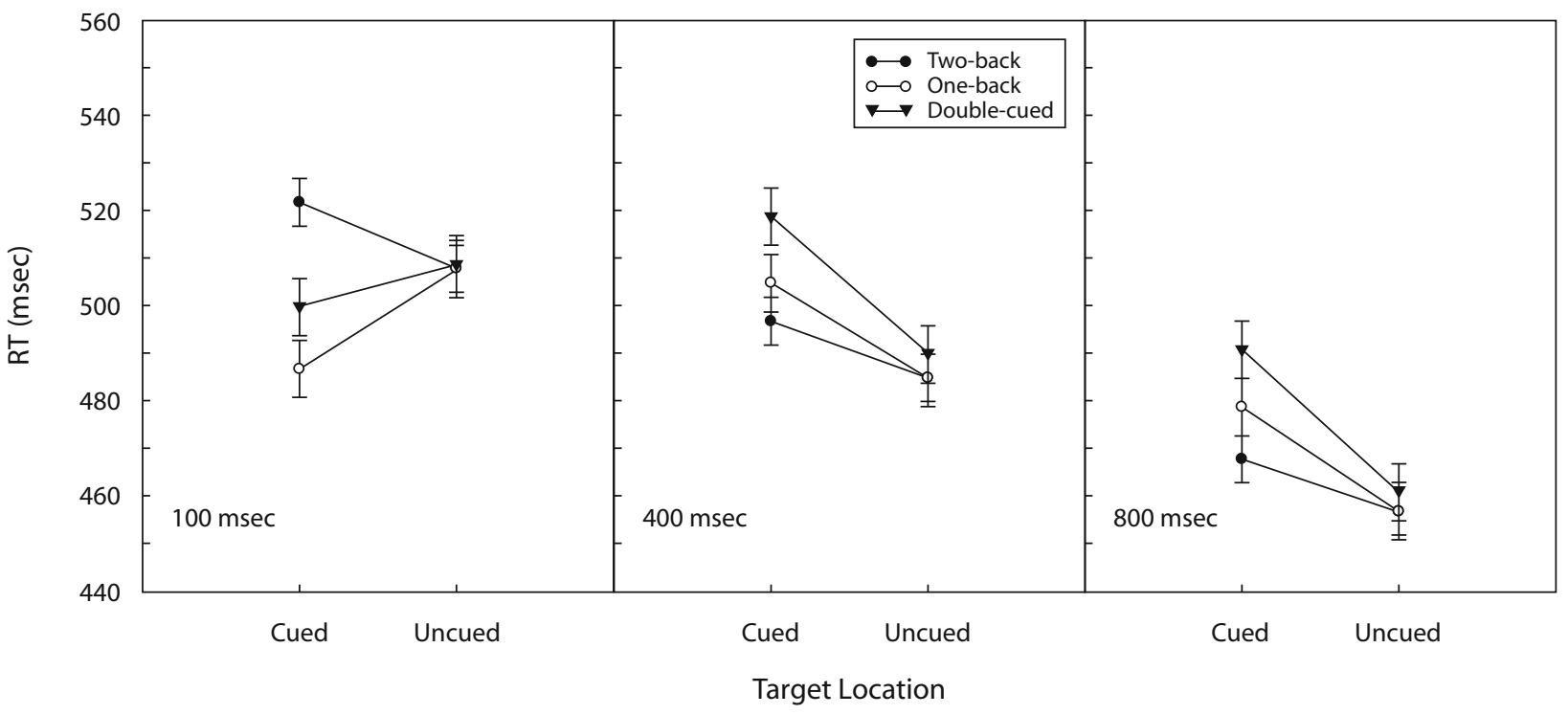

Figure 4. Mean target response times (RTs, in milliseconds) on correct trials, as a function of whether targets were presented at cued or uncued locations in Experiment 4. Numbers in the bottom-left corner of each graph represent the second cue-target SOA for that condition. "Two-back" refers to trials on which targets appeared in the first location cued; "one-back" refers to trials on which targets appeared in the second location cued; "double-cued" refers to trials on which targets appeared at a location cued twice. Note that two-back and one-back uncued trials are identical. Error bars represent within-participants $95 \%$ confidence intervals calculated using the methodology outlined by Masson and Loftus (2003).

main effects of SOA $\left[F(2,46)=59.45, p<.001, \eta^{2}=\right.$ $.721]$ and validity $\left[F(1,23)=24.26, p<.001, \eta^{2}=.513\right]$, but no interaction between these factors $[F(2,46)=0.26$, $\left.p>.77, \eta^{2}=.011\right]$. As in Experiment 3, it is clear that the main effect of SOA stems from a decline in overall RTs with increasing SOA. To examine the main effect of validity, we compared RTs for cued and uncued targets at each SOA. These comparisons revealed IOR magnitudes of $14 \mathrm{msec}$ at the $100-\mathrm{msec} \mathrm{SOA}[t(23)=3.55, p<.01]$, $12 \mathrm{msec}$ at the $400-\mathrm{msec} \mathrm{SOA}[t(23)=3.24, p<.01]$, and $11 \mathrm{msec}$ at the $800-\mathrm{msec}$ SOA $[t(23)=3.46, p<$ .01]. Finally, it is also notable that overall RTs were much slower in Experiment 4 than in the earlier experiments. This is consistent with the results of other studies that have used localization tasks (e.g., Prime, Visser, \& Ward, 2006) and presumably reflects the greater difficulty of the targetlocalization task relative to the target-detection task used in Experiments 1-3.

To examine the impact of SOA in the one-back condition, we submitted the relevant means to an identical $3(\mathrm{SOA}) \times 2$ (validity) within-participants ANOVA. This revealed significant main effects of SOA $[F(2,46)=$ $\left.23.29, p<.001, \eta^{2}=.503\right]$ and validity $[F(1,23)=4.64$, $\left.p<.05, \eta^{2}=.168\right]$, as well as an interaction between these factors $\left[F(2,46)=32.00, p<.001, \eta^{2}=.582\right]$. As in the two-back condition, the main effect of SOA reflects a decline in overall RTs as SOA increased. To examine the interaction, we compared RTs for cued and uncued targets at each SOA. These comparisons revealed a facilitatory effect of $21 \mathrm{msec}$ at the 100-msec SOA [ $t(23)=$ $4.80, p<.001]$ and IOR magnitudes of $20 \mathrm{msec}$ at the 400 -msec SOA $[t(23)=4.19, p<.001]$ and $22 \mathrm{msec}$ at the $800-\mathrm{msec}$ SOA $[t(23)=4.38, p<.001]$.
Finally, to examine the impact of second cue-target SOA in the double-cued condition, we submitted the relevant means to a $3(\mathrm{SOA}) \times 2$ (validity) within-participants ANOVA. This revealed significant main effects of SOA $\left[F(2,46)=21.59, p<.001, \eta^{2}=.484\right]$ and validity $\left[F(1,23)=28.37, p<.001, \eta^{2}=.552\right]$, as well as an interaction between these factors $[F(2,46)=29.23, p<$ $\left..001, \eta^{2}=.560\right]$. Once again, the main effect of SOA reflects the fact that overall RTs decreased with an increase in SOA. To examine the interaction, we compared RTs for cued and uncued targets at each SOA. These comparisons revealed a facilitatory effect of $9 \mathrm{msec}$ at the 100 -msec SOA $[t(23)=1.80, p<.05$, one-tailed $]$ and IOR magnitudes of $30 \mathrm{msec}$ at the $400-\mathrm{msec} \mathrm{SOA}[t(23)=6.15, p<$ $.001]$ and $31 \mathrm{msec}$ at the $800-\mathrm{msec}$ SOA $[t(23)=6.97$, $p<.001]$.

As in Experiment 3, we tested two predictions made by the hypothesis that successive cues would have summative effects on target performance. First, we examined whether, in the double-cued condition, less IOR would be found at the 100-msec second cue-target SOA than at the 800 -msec SOA. This prediction was confirmed by the data $[t(23)=6.43, p<.001]$. Next, we expected to find less IOR at double-cued locations than at two-back locations at the $100-\mathrm{msec}$ SOA. This prediction was confirmed by the data as well $[t(23)=3.93, p<.01]$.

Finally, we compared the magnitude of facilitation/ IOR between the double-cued and one-back conditions at each second cue-target SOA. At the 100-msec SOA, there was significantly more facilitation in the one-back condition $[t(23)=2.07, p=.05]$. At the 400-msec SOA, there was significantly greater IOR in the double-cued condition $[t(23)=2.20, p<.04]$. At the 800 -msec SOA, 
there was also significantly greater IOR in the doublecued condition $[t(26)=1.88, p<.04$, one-tailed]. These results are consistent with the notion that cuing a location twice yields a summation of the effects of both cues on target localization. ${ }^{1}$

In comparing the findings of Experiments 3 and 4, it is clear that a substantially similar pattern of results occurred. However, there were some differences. Certainly, overall RTs were considerably longer in all conditions. In addition, there was greater facilitation in both the oneback and double-cued conditions at the 100-msec SOA. This is consistent with suggestions by Klein (2000) that more complex tasks, such as localization and discrimination, can lead to stronger facilitation and delayed onset of IOR (see also, Lupiáñez, Milliken, Solano, Weaver, \& Tipper, 2001) due to increased dwell time of attention.

\section{GENERAL DISCUSSION}

According to the forage facilitator hypothesis (Klein, 1988, 2000; Klein \& MacInnes, 1999), IOR reflects underlying inhibitory mechanisms designed to prevent reinspection of spatial locations that have already been searched without success. Consistent with this notion, Danziger et al. (1998) found that IOR dwelled at three different cued locations, implying that inhibitory mechanisms could operate simultaneously at multiple spatial locations. One issue that has received less consideration, however, is how the visual system responds to multiple occurrences of irrelevant events at the same spatial location.

The present work investigated this issue using a cuing paradigm in which two cues were presented in either the same or different locations prior to target onset. The goal was to compare the magnitude of IOR at a spatial location, as a function of number of prior cues. In Experiments 1 and 2, we showed that cuing a location twice resulted in greater IOR than did cuing a location once. This finding clarified earlier results obtained by Pratt and Abrams (1995) and Maylor and Hockey (1987) and was replicable across changes in numbers of potential target locations and their spatial configuration. In Experiments 3 and 4, we showed evidence consistent with the notion that presentation of the second cue initiated a cycle of facilitation followed by inhibition similar to that which occurs when a cue is presented at a novel location. These effects of the second cue roughly summated with those of the first cue, generating overall facilitation when the second cue-target interval was brief and enhancing inhibition when the second cue-target interval was longer. In addition, the results of Experiment 4 showed that the influence of double cuing extended from simple detection tasks to a more complex localization task.

A key question that motivated this work concerned the response of the visual system to distraction. IOR studies suggest that irrelevant visual events, including nonpredictive visual cues, can lead to the inhibition of visual (Handy et al., 1999), oculomotor (Rafal, Calabresi, Brennan, \& Sciolto, 1989), and response (Ivanoff \& Klein, 2001) types of processing. Such work has typically focused on a single distractor presented prior to target onset or at suc- cessive irrelevant events presented in a very sparse visual environment (Pratt \& Abrams, 1995). In the real world, however, distracting events at a single location often occur on multiple occasions in more complex environments. For example, flashing lights on a movie billboard repeatedly generate visual transients, or, as in an earlier example, a stranger may repeatedly wave to another person.

On one hand, it is reasonable to conjecture that multiple irrelevant events would not generate additional inhibition. That is, having inhibited a location on one occasion, this may be sufficient to prevent reexamination, even in the face of additional irrelevant events. An additional advantage to this option is that it leaves more resources available should irrelevant events at other spatial locations require inhibition. On the other hand, it is equally reasonable that continued salient events at a given spatial location should yield more inhibition. Given the very real possibility that these events could lead to fruitless reexamination of a searched location, it may be desirable to increase inhibition to prevent this from happening.

The present results suggest that when faced with multiple irrelevant events, the visual system opts for the latter option and places additional inhibition at a previously cued location. In fact, it would seem that a nonpredictive visual event is treated largely the same, regardless of its spatial location. That is, it will generate a period of facilitation, possibly reflecting the beneficial effects of transient visual attention being drawn to the cued location, followed by inhibition. Indeed, examination of the data shows that similar levels of enhanced inhibition in the double-cued condition were found across number of potential target locations (4 vs. 3, Experiment 1 vs. Experiment 2 ) and across target task (detection vs. localization, Experiments 2 and 3 vs. Experiment 4).

This relatively intransient response to multiple cues presented at the same spatial location may be surprising in the face of suggestions that IOR reflects an adaptive response to environmental properties. For example, Klein (2000) argued that IOR is at least partly a learned behavior derived from past visual search experience, whereas Tipper et al. (1996) advanced the notion that such inhibition stems from the application of flexible biological mechanisms, and Hunt and Kingstone (2003) suggested that IOR reflects multiple inhibitory mechanisms that are recruited to greater or lesser degrees, depending on task parameters. Consistent with such suggestions, whereas multiple-location IOR has not been observed in twolocation paradigms, where it would not be desirable to inhibit both locations (e.g., Pratt \& Abrams, 1995), it has been observed repeatedly in paradigms with four or more locations (e.g., Danziger et al., 1998), where inhibiting more than one location simultaneously would make search more efficient.

However, closer consideration of the present results suggests that treating each instance of a cue similarly, regardless of whether it occurs at a novel or old location, may actually reflect optimal adaptation to the environment. Arguably, when two separate visual events occur at the same location, it is desirable to reexamine this location because the new visual event likely reflects a potentially 
important change in the environment. Then, if this reexamination proves to be unnecessary (because no object of interest is present), it may be equally desirable to place inhibition at that spatial location, even if some inhibition is already present, in order to deter future fruitless searches. This follows from the reasonable assumption that the probability of an important visual event occurring at a location decreases as the number of irrelevant events occurring there increases (this may not be statistically accurate, since visual events may be independent, but it is likely an excellent heuristic). Of course, this argument implies that the systems underlying IOR are sensitive to sequential probabilities of events in the environment and can apply this knowledge. This suggestion is broadly consistent with studies showing that empirical conditions leading to IOR change with age (e.g., Brodeur \& Enns, 1997; MacPherson, Klein, \& Moore, 2003), implying that visual search mechanisms may vary with exposure to the environment.

Another theoretically notable aspect of our results concerns the relationship between facilitatory and inhibitory effects of cues. Since the seminal work of Posner and Cohen (1984), it is been assumed that the presence of visual attention at a spatial location would counteract the influence of IOR. This has given rise to the relatively common practice of inserting a reorienting event (the presentation of a visual transient at fixation) between cues and targets in IOR experiments (see Prime et al., 2006). This visual transient is thought to draw attention resources to its location and away from the location of the cue.

The assumption that visual attention counteracts inhibition is consistent with evidence that predictive visual cues, whether endogenous or exogenous, do not generate IOR (e.g., Jonides \& Irwin, 1981; Nakayama \& Mackeben, 1989; Posner, 1980), as well as the common finding that presentation of an irrelevant peripheral event generates facilitation followed by inhibition (e.g., Posner \& Cohen, 1984). That said, a number of experiments have failed to obtain early facilitation from nonpredictive peripheral cues (e.g., Prime et al., 2006; Riggio, Bello, \& Umiltà, 1998; Tassinari, Aglioti, Chelazzi, Peru, \& Berlucchi, 1994), even at brief cue-target intervals. The absence of early facilitation implies that at visual attention, which was presumably attracted when the cue was presented, was not effective in counteracting the impact of inhibitory processes also arising from the cue's presentation.

The present work offers a unique opportunity to examine this issue, because we presented successive cues at the same spatial location and thus could directly examine the impact of visual attention (attracted by the second cue) on inhibitory processes already initiated by an initial cue. The results from these experiments are clear. In Experiments 3 and 4, when targets were presented 800 msec after the onset of an initial nonpredictive cue, significant IOR was found. On the other hand, in the nearly identical case in which targets followed an initial cue by $900 \mathrm{msec}$, but were also preceded by a second cue at the same location $100 \mathrm{msec}$ earlier, facilitation was found. This provides the first direct evidence in the context of a visual cuing paradigm typically used in IOR experiments that the facili- tatory effects of visual attention can counteract the inhibitory effects generated by a nonpredictive cue.

With respect to implications for theoretical accounts of IOR, much debate has occurred about whether inhibition is generated immediately on the presentation of a nonpredictive cue or whether inhibition does not begin until attention leaves the location of the cue (e.g., Chica, Lupiáñez, \& Bartolomeo, 2006; Danziger \& Kingstone, 1999). The present work does not allow us to distinguish between these alternatives directly. However, our findings that the facilitatory effects of visual cues can ameliorate inhibition generated by prior cues suggest that it is entirely plausible that early facilitatory effects of visual attention mask existing inhibition generated on presentation of a visual cue.

\section{Conclusion}

The present work demonstrates that multiple cues presented in the same spatial location have cumulative effects on target detection and localization. This addresses an important question directly applicable to complex world scenes that often feature multiple irrelevant onsets presented at the same spatial location. However, a number of important questions remain to be answered by future research. First, whereas it may be tacitly assumed that peripheral visual cues presented here attracted visual attention, it is unclear whether oculomotor mechanisms also have played a role in our pattern of results. This is because we were unable to monitor eye movements to ensure maintenance of fixation. That our results did not differ significantly regardless of whether fixation was removed prior to a trial (Experiment 1) or remained onscreen (Experiments 2-4) suggests that oculomotor mechanisms play a minimal role. However, this is a relatively weak test of this question. Therefore, it is crucial that future studies investigate the role of oculomotor mechanisms in generating IOR when a single location is cued on multiple occasions. Second, whereas the present findings suggest that the effects of multiple cues remain invariant across different numbers of locations and tasks, it would be desirable to explore this relationship parametrically, considering more than two cues and four locations. Also relevant would be an examination of more ecologically valid search environments. Taken together, such work would likely add important additional information to our growing body of knowledge about the processing of visual distractors.

\section{AUTHOR NOTE}

The first author is now at the University of Queensland. This work was sponsored by a research grant from the Natural Sciences and Engineering Research Council of Canada. The authors thank Jessica Jackson and Sarah Barnes for assistance in data collection, as well as Mike Dodd, Angus Gellatly, and an anonymous reviewer for thoughtful comments on an earlier version of this article. Correspondence concerning this article should be addressed to T. A. W. Visser, School of Psychology, University of Queensland, St. Lucia, QLD, 4072, Australia (e-mail: t.visser@ uq.edu.au).

\section{REFERENCES}

Abrams, R. A., \& Pratt, J. (1996). Spatially diffuse inhibition affects 
multiple locations: A reply to Tipper, Weaver, and Watson (1996). Journal of Experimental Psychology: Human Perception \& Performance, 22, 1294-1298.

Birmingham, E., \& Pratt, J. (2005). Examining inhibition of return with onset and offset cues in the multiple-cuing paradigm. Acta Psychologica, 118, 101-121.

Brodeur, D. A., \& EnNs, J. T. (1997). Covert visual orienting across the life span. Canadian Journal of Experimental Psychology, 51, 20-35.

Chica, A. B., Lupiáñez, J., \& Bartolomeo, P. (2006). Dissociating inhibition of return from endogenous orienting of spatial attention: Evidence from detection and discrimination tasks. Cognitive Neuropsychology, 23, 1015-1034.

Coward, R. S., Poliakoff, E., O'Boyle, D. J., \& Lowe, C. (2004). The contribution of nonocular response inhibition to visual inhibition of return. Experimental Brain Research, 155, 124-128.

Danziger, S., \& Kingstone, A. (1999). Unmasking the inhibition of return phenomenon. Perception \& Psychophysics, 61, 1024-1037.

Danziger, S., Kingstone, A., \& SNyder, J. J. (1998). Inhibition of return to successively stimulated locations in a sequential visual search paradigm. Journal of Experimental Psychology: Human Perception \& Performance, 24, 1467-1475.

DodD, M. D., Castel, A. D., \& Pratt, J. (2003). Inhibition of return with rapid serial shifts of attention: Implications for memory and visual search. Perception \& Psychophysics, 65, 1126-1135.

FernandeZ-Duque, D., \& Posner, M. I. (1997). Relating the mechanisms of orienting and alerting. Neuropsychologia, 35, 477-486.

Gellatly, A., Cole, G., Fox, C., \& Johnson, M. (2003). Response inhibition can affect reaction time to abrupt-onset visual displays. Perception, 32, 1377-1391.

Handy, T. C., Jha, A. P., \& Mangun, G. R. (1999). Promoting novelty in vision: Inhibition of return modulates perceptual-level processing. Psychological Science, 10, 157-161.

Harvey, N. (1980). Non-informative effects of stimuli functioning as cues. Quarterly Journal of Experimental Psychology, 32, 413-425.

Hunt, A. R., \& Kingstone, A. (2003). Inhibition of return: Dissociating attentional and oculomotor components. Journal of Experimental Psychology: Human Perception \& Performance, 29, 1068-1074.

Ivanoff, J., \& KLEIN, R. M. (2001). The presence of a nonresponding effector increases inhibition of return. Psychonomic Bulletin \& Review, 8 , 307-314

Jonides, J., \& Irwin, D. E. (1981). Capturing attention. Cognition, 10, 145-150.

KLEIN, R. M. (1988). Inhibitory tagging system facilitates visual search. Nature, 334, 430-431.

KLEIN, R. M. (2000). Inhibition of return. Trends in Cognitive Sciences, 4, 138-147.

KLEIN, R. M., \& MACInNES, W. J. (1999). Inhibition of return is a foraging facilitator in visual search. Psychological Science, 10, 790-801.

Lupiáñez, J., Milliken, B., Solano, C., Weaver, B., \& Tipper, S. P. (2001). On the strategic modulation of the time course of facilitation and inhibition of return. Quarterly Journal of Experimental Psychology, 54A, 753-773.

MacPherson, A. C., Klein, R. M., \& Moore, C. (2003). Inhibition of return in children and adolescents. Journal of Experimental Child Psychology, 85, 337-351.

Masson, M. E. J., \& LofTUs, G. R. (2003). Using confidence intervals for graphically based data interpretation. Canadian Journal of Experimental Psychology, 57, 203-220.

MAYLOR, E. A., \& HocKeY, R. (1987). Effects of repetition on the facilitatory and inhibitory components of orienting in visual space. Neuropsychologia, 25, 41-54.
Nakayama, K., \& Mackeben, M. (1989). Sustained and transient components of focal visual attention. Vision Research, 29, 1631-1647.

OgaWA, H., TAKedA, Y., \& YAGI, A. (2002). Inhibitory tagging on randomly moving objects. Psychological Science, 13, 125-129.

PAul, M. A., \& TiPPER, S. P. (2003). Object-based representations facilitate memory for inhibitory processes. Experimental Brain Research, 148, 283-289.

Posner, M. I. (1980). Orienting of attention. Quarterly Journal of Experimental Psychology, 32, 3-25.

Posner, M. I., \& Cohen, Y. (1984). Components of visual orienting. In H. Bouma \& D. G. Bouwhuis (Eds.), Attention and performance X: Control of language processes (pp. 531-556). Hillsdale, NJ: Erlbaum.

Pratt, J., \& Abrams, R. A. (1995). Inhibition of return to successively cued spatial locations. Journal of Experimental Psychology: Human Perception \& Performance, 21, 1343-1353.

Pratt, J., \& Chasteen, A. L. (2007). Examining inhibition of return with multiple sequential cues in younger and older adults. Psychology \& Aging, 22, 404-409.

Prime, D. J., Visser, T. A. W., \& WARD, L. M. (2006). Reorienting attention and inhibition of return. Perception \& Psychophysics, $\mathbf{6 8}$ 1310-1323.

Rafal, R. D., Calabresi, P. A., Brennan, C. W., \& Sciolto, T. K. (1989). Saccade preparation inhibits reorienting to recently attended locations. Journal of Experimental Psychology: Human Perception \& Performance, 15, 673-685.

RigGio, L., Bello, A., \& Umiltà, C. (1998). Inhibitory and facilitatory effects of cue onset and offset. Psychological Research, 61, 107-118.

Snyder, J. J., \& Kingstone, A. (2000). Inhibition of return and visual search: How many separate loci are inhibited? Perception \& Psychophysics, 62, 452-458.

SNYDER, J. J., \& KingSTONE, A. (2001). Inhibition of return at multiple locations in visual search: When you see it and when you don't. Quarterly Journal of Experimental Psychology, 54A, 1221-1237.

SNydER, J. J., \& KingSTONE, A. (2007). Inhibition of return at multiple locations and its impact on visual search. Visual Cognition, 15, 238256

Tassinari, G., Aglioti, S., Chelazzi, L., Peru, A., \& Berlucchi, G. (1994). Do peripheral noninformative cues induce early facilitation of target detection? Vision Research, 34, 179-189.

Tipper, S., Weaver, B., \& Watson, F. (1996). Inhibition of return to successively cued spatial locations: Commentary on Pratt and Abrams (1995). Journal of Experimental Psychology: Human Perception \& Performance, 22, 1289-1293.

\section{NOTE}

1. It is notable that in both Experiments 3 and 4, the magnitude of IOR in the two-back condition when cue-target SOA is $900 \mathrm{msec}$ is significantly less than the magnitude of IOR in the one-back condition when cue-target SOA is $800 \mathrm{msec}$, despite having nearly identical cuetarget intervals. We suggest that this difference might reflect two factors: spreading inhibition from the location of the first cue to that of the second cue in the one-back condition, and spreading facilitation from the location of the second cue to the location of the first cue in the twoback condition. However, this explanation is post hoc, and is in need of empirical validation.

(Manuscript received April 7, 2008; revision accepted for publication September 10, 2008.) 